\title{
Matrix Metalloproteinase 3: A Promoting and Destabilizing Factor in the Pathogenesis of Disease and Cell Differentiation
}

\author{
Jiangtao Wan', Guowei Zhang', Xin Li1, Xianshuai Qiu', Jun Ouyang', Jingxing Dai2* \\ and Shaoxiong Min ${ }^{1 *}$
}

${ }^{1}$ Spine Surgery, Zhujiang Hospital, Southern Medical University, Guangzhou, China, ${ }^{2}$ Guangdong Provincial Key Laboratory of Medical Biomechanics, Department of Anatomy, School of Basic Medical Sciences, Southern Medical University,

Guangzhou, China

\section{OPEN ACCESS}

Edited by:

Jue Wang,

The University of Texas Health Science Center at Tyler, United States

Reviewed by:

Antonio Gaballo,

National Research Council (CNR), Italy

Jun Fan,

China Medical University, China

${ }^{*}$ Correspondence:

Jingxing Dai

daijx@smu.edu.cn

Shaoxiong Min

msxbear24@163.com

Specialty section:

This article was submitted to

Lipid and Fatty Acid Research,

a section of the journal

Frontiers in Physiology

Received: 04 February 2021

Accepted: 10 June 2021

Published: 02 July 2021

Citation:

Wan J, Zhang G, Li X, Qiu X, Ouyang J, Dai J and Min S (2021)

Matrix Metalloproteinase

3: A Promoting and Destabilizing Factor in the Pathogenesis of Disease and Cell Differentiation.

Front. Physiol. 12:663978.

doi: 10.3389/fphys.2021.663978
Cells must alter their expression profiles and morphological characteristics but also reshape the extracellular matrix (ECM) to fulfill their functions throughout their lifespan. Matrix metalloproteinase 3 (MMP-3) is a member of the matrix metalloproteinase (MMP) family, which can degrade multiple ECM components. MMP-3 can activate multiple proMMPs and thus initiates the MMP-mediated degradation reactions. In this review, we summarized the function of MMP-3 and discussed its effects on biological activities. From this point of view, we emphasized the positive and negative roles of MMP3 in the pathogenesis of disease and cell differentiation, highlighting that MMP-3 is especially closely involved in the occurrence and development of osteoarthritis. Then, we discussed some pathways that were shown to regulate MMP-3. By writing this review, we hope to provide new topics of interest for researchers and attract more researchers to investigate MMP-3.

Keywords: matrix metalloproteinase 3, osteoarthritis, cell differentiation, stem cells, extracellular matrix

\section{INTRODUCTION}

Matrix metalloproteinase-3 (MMP-3), also known as stromelysin-1, is a member of the matrix metalloproteinase family (MMPs). The function of this molecule depends on zinc ions in the internal environment. MMP-3 is composed of $475-478$ amino acids in mammals, and its amino acid sequence is highly conserved among different species and genera, suggesting its potential for cross-species applications ${ }^{1}$. Like that of most MMPs, the structure of MMP-3 can be divided into four parts: a propeptide of approximately 80 amino acids, a metalloproteinase catalytic domain of approximately 170 amino acids, a ligating peptide of variable length (hinged region) and a heme protein domain of approximately 200 amino acids. MMP-3 can digest a variety of extracellular matrix (ECM) components, including matrix proteins, growth factors, proteases, surface receptors, and adhesion molecules. In particular, this enzyme can process a variety of pro-MMPs, and thus, the synthesis and activation of MMP-3 the first step in the MMP-mediated degradation process (Nagase et al., 2006; Pinto et al., 2006; Inge et al., 2012; Cui et al., 2017); furthermore, this molecule is associated with cellular fibrinolytic activity (Lijnen, 2002). Tissue inhibitor of metalloproteinase

${ }^{1}$ www.brenda-enzymes.org 
(TIMP) is a natural MMP inhibitor. As early as 2003, researchers proposed that the balance between MMPs and TIMPs is essential for the integrity of the ECM and that the proteolytic changes mediated by these molecules may lead to a variety of pathological states (Carine et al., 2003).

Matrix metalloproteinase 3 is an exocrine protein that is usually secreted through exocytosis and extracellular vesicles (EVs). Researchers extracted the exosomes of interleukin-1 beta (IL-1 $\beta$ )-treated synovial fibroblasts and detected MMP-3 in these exosomes, although the level was low (Kato et al., 2014). Another study found that adipose stem cells (ASC)-derived exosomecontaining medium reduced tumor necrosis factor alpha (TNF$\alpha$ )-induced osteocyte hypertrophy, and this effect was dependent on the abundance of MMPs and TIMPs in the exosomes (Niada et al., 2019). A previous study analyzed cytokines within ASCderived exosomes to determine the cause of their chondrocyteprotective function. These researchers found high levels of TIMPs but barely any MMPs in the ASC-derived exosomes (Woo et al., 2020). Studies on the MMP-3 level in EVs are currently limited, and more efforts are needed to reveal the conditions in which MMP-3 can be secreted, taken up and function through EVs. MMP-3 can also be found in the nucleus and participate in the transcriptional process of chondrocytes separated from chondromas (Eguchi et al., 2008).

Current studies on MMP-3 mainly focus on tumor migration and central nervous system (CNS) development, such as axon growth and remodeling (Andries et al., 2017). In fact, as a stromal shaping agent, MMP-3 is widely involved in multiple cell biological processes, such as cell differentiation and inflammation. Given the extensive biological functions of MMP3 and its involvement in a variety of diseases, this molecule can be applied as an indicator and therapeutic target of diseases. In the following sections, we summarize the biological functions and clinical applications of MMP-3 based on existing studies, hoping to provide new topics of interest for researchers and attract more researchers to investigate MMP-3.

\section{MMP-3 EXTENSIVELY PARTICIPATES IN CELL DIFFERENTIATION AND DISEASE PROGRESSION}

\section{Cell Differentiation}

During differentiation, in addition to changes in the cell expression profile, an appropriate ECM environment is needed to sustain this process. In 1997, researchers proposed that MMP3 expression is tissue-specific during morphogenesis of the rat maxillofacial region; that is, MMP-3 is expressed only in the region of mandibular ossification (Chin and Werb, 1997). Later, after analysis of the tissues of the lower jaw of patients with mandibular hypoplasia, researchers confirmed that MMP-3 genetic abnormalities can lead to mandibular hypoplasia. Based on in vitro experiments, BMAL1 defects induced by activating p65 phosphorylation were shown to enhance the transcription of MMP-3 and then inhibit osteogenic differentiation and promote osteoclast maturity, causing a decrease in bone mass, and eventually leading to mandibular hypoplasia (Jiajia et al., 2018). Researchers used antibody array analysis to study the cytokines secreted by stem cells derived from different tissues and concluded that MMP-3 expression levels differ between stem cells from exfoliated deciduous teeth (SHEDs) and bone marrow mesenchymal stem cells. These researchers also found that most stem cells constantly express MMP-3, suggesting that MMP-3 may play an important role in maintenance of stemness or differentiation (Yamada et al., 2019). Bone Morphogenetic Protein (BMP-4) can reduce the expression levels of both MMP-3 and MMP-13, reduce extracellular proteoglycan and collagen degradation, and finally inhibit C3H10T1/2 stem cell differentiation into adipocytes (Otto et al., 2007). Moreover, by observing and monitoring the changes in the levels of MMP-3 and TIMP-1 expressed by breast adipocytes during the lactation period, researchers found that MMPs, especially MMP-3, are involved in the proliferation and hypertrophy of adipocytes. They further confirmed this theory both in vivo and in vitro with 3T3-L1 cell lines (Alexander et al., 2001).

Noggin can promote the differentiation of mouse-derived embryonic stem cells into cardiomyocytes in a MMP-3dependent manner (Hong et al., 2010). In the nervous system, transitional cells differentiated from adult neuroprogenitor cells (aNPCs) expressed increased levels of MMP-3 and MMP-9 under the effect of stromal cell-derived factor 1 and vascular endothelial growth factor. The above process can be interrupted by blocking the expression of MMP-3 or MMP-9 in aNPCs (Barkho et al., 2008). Axon inhibition is a major obstacle to nerve regeneration in adult mammals. Some researchers speculated that MMP-3 could abolish axon inhibition and then promote nerve regeneration by destroying the stromal layer and glial scar around neurons (Andries et al., 2017). Analyses of human embryogenesis and development further indicated that MMP-3 participates in the regulation of cell differentiation. RT-PCR, Western blots and enzyme activity analyses showed that the precursor and active forms of MMP-3 were expressed in villous trophoblasts during early pregnancy, differentiated invasive cytotrophoblasts and trophoblast SGHPL-4 cells (Husslein et al., 2009), suggesting that MMP-3 may play a role in early embryogenesis and germ layer differentiation.

\section{Disease Progression}

Matrix metalloproteinase 3 has many substrates, and thus, it is involved in the occurrence and development of various diseases (Figure 1). MMP-3 can degrade ECM, which is beneficial for tumor invasion and metastasis. Researchers compared MMP-3 mRNA levels between tumor cells and osteoblast cells in patients with osteosarcoma and concluded that osteosarcoma tumor cells express more MMP-3. Subsequent knockout experiments showed that MMP-3 knockout can reduce the migration of tumor cells, which confirmed that MMP-3 contributes to osteosarcoma invasion (Huang J. F. et al., 2016). Similar conclusions were reported by other researchers who inhibited the expression of MMP-3 with miR-134 (Chen et al., 2019). Moreover, other studies on ovarian cancer (Zheng et al., 2019) and anaplastic thyroid tumors (Ma et al., 2019) have reported the same effect of MMP-3. 


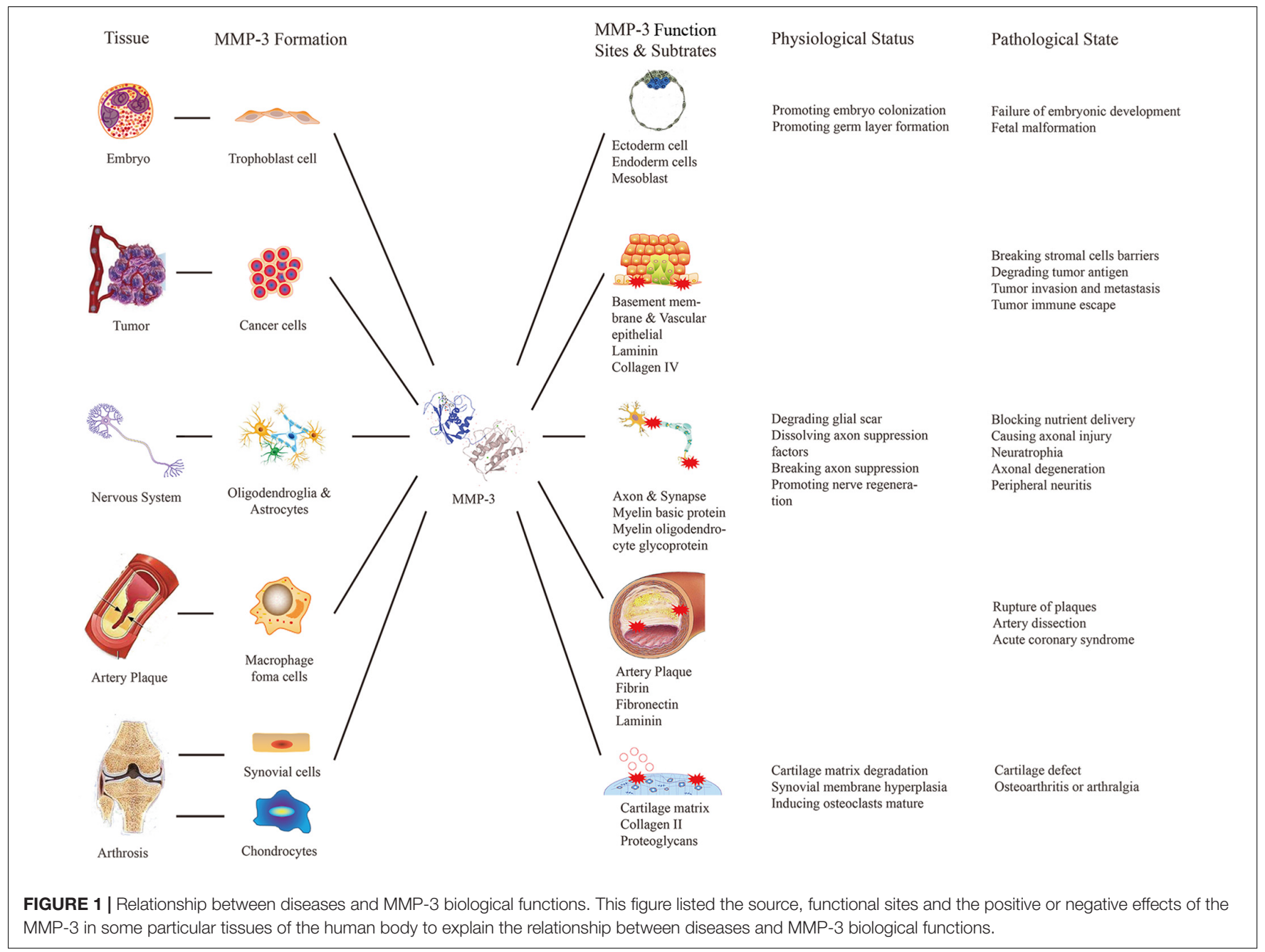

Matrix metalloproteinase 3 is considered a common inflammatory factor that can promote inflammation in different parts of the body. In the nervous system, the typical pathological changes are extensive inflammation and axonal injury. Compared with that in the CNS, peripheral nerve injury, such as optic atrophy, macular degeneration numbness and hypesthesia, is more common (De Groef et al., 2015). Peripheral nerve injury caused by paclitaxel is thought to be related to excessive MMP-3 secretion (Nishida et al., 2008). However, the role of MMP-3 in the nervous system is complicated, and its ability to degrade ECM is considered to be a key factor in the disruption of the inhibitory CNS environment in the early stage of nerve regeneration (Andries et al., 2017). In the cardiovascular system, pathological changes caused by MMP-3 include endothelial injury and inflammatory cell accumulation. Studies have confirmed that the sustained expression of MMP-3 is closely related to atherosclerotic plaque rupture (Huang et al., 2017) because MMP-3 can interact with plasminogen and fibrinogen, showing antithrombotic activity. Some researchers have proposed that MMP-3 can be used to monitor plaque stability (Guizani et al., 2019) or as a target for preventing plaque rupture. Additionally, MMP-3 gene polymorphisms are closely related to the occurrence and development of cardiovascular diseases such as atherosclerosis and rheumatic heart disease (Huang X. Y. et al., 2016; Ghaffarpour et al., 2017; Hu et al., 2018). In musculoskeletal system, MMP-3 abnormalities can lead to pathological changes such as cartilage matrix degradation and synovial hyperplasia. High expression levels of MMP-1, MMP-2, MMP-3, MMP-8, MMP-9, and MMP-13 were found in the meniscus after inflammation or injury. The MMP-3 level in synovial fluid increased 30-40 times, while the level of TIMP-1 increased 10-fold within $24 \mathrm{~h}$ after meniscal injury (Lee et al., 2016).

\section{MMP-3 IS CLOSELY INVOLVED IN THE OCCURRENCE AND DEVELOPMENT OF OSTEOARTHRITIS}

Osteoarthritis (OA) is one of the most common degenerative diseases, and the prevalence of knee $\mathrm{OA}$ alone has reached $3.8 \%$, affecting more than 250 million people worldwide (Cross et al., 2014). The typical pathological change in OA is 


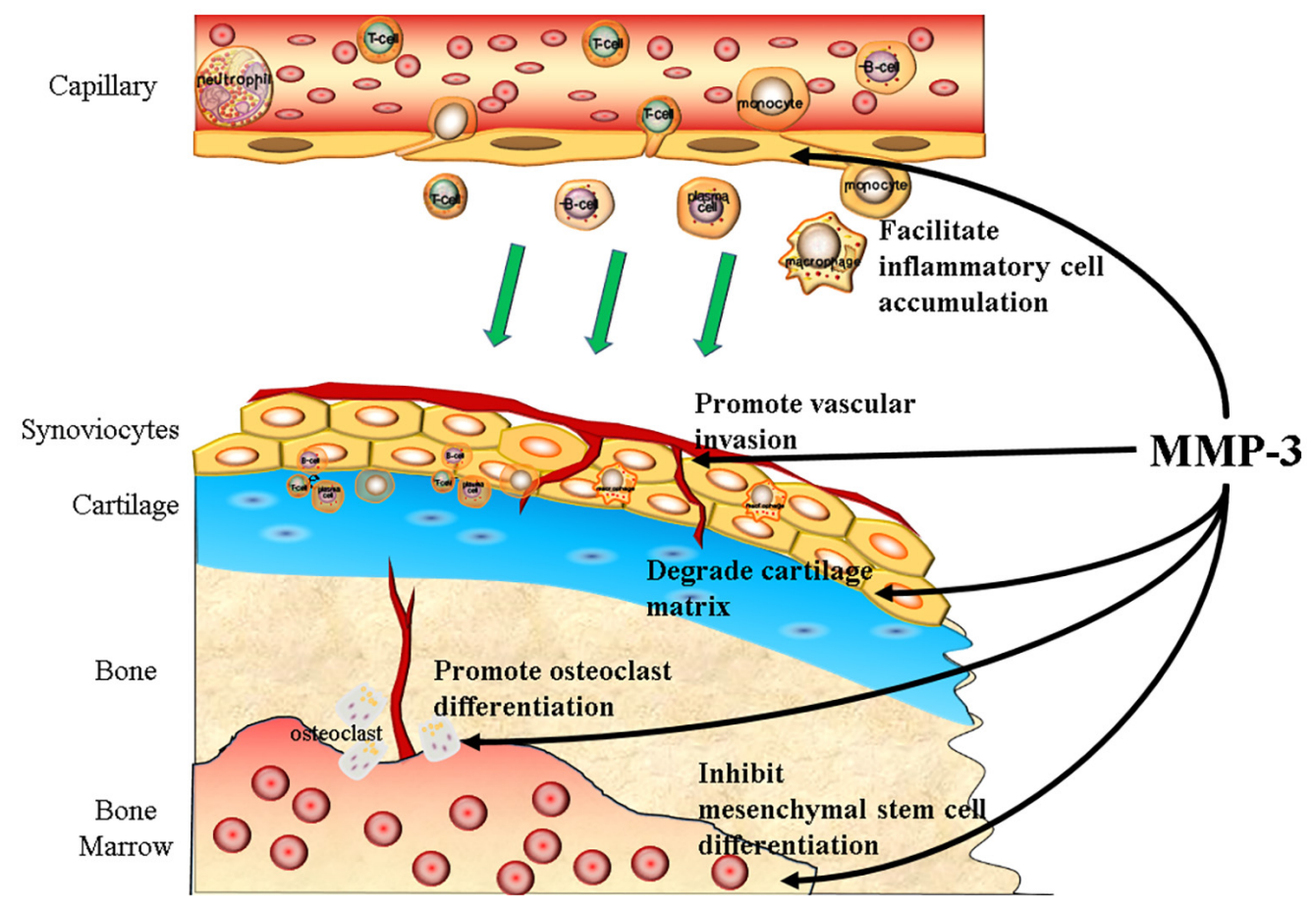

FIGURE 2 | MMP-3 participates in the progression of OA. MMP-3 can promote the progression of OA both in preclinical and clinical stage. To be precisely, MMP-3 can facilitate the inflammatory cells accumulation, promote vascular invasion, degrade cartilage matrix, promote osteoclast differentiation, and inhibit mesenchymal stem cells differentiation.

cartilage degradation, and thus, MMP-3 participates in almost every step of the progression of OA (Figure 2).

At the preclinical stage of OA, MMP-3 levels increase in response to axial compression and degradation of cartilage matrix together with MMP-13 and ADAMTS-4 (Behrendt et al., 2016), while low mechanical load can reduce the release of MMP-3 and cartilage damage (He et al., 2020). Moreover, some particular MMP-3 genetic variation can lead to susceptibility to OA, according to a cross-sectional study including 431 females (Tong et al., 2017).

At the clinical stage of OA, MMP-3 can promote vessel growth into cartilage, facilitate inflammatory cell accumulation and inhibit mesenchymal stem cells from differentiating into chondrocytes. Vascular invasion is one of the signs of cartilage degeneration, and evidence has shown that MMP-3 participates in vascular formation (Chen et al., 2013). Normal cartilage does not have high levels of protease inhibitors (Franses et al., 2010), indicating it can be resistant to high MMP levels. The MMP-3 expression level in synovial cells is higher than that in cartilage, and slight angiogenesis is observed in the knee of OA patients (Koyama et al., 2020). Vascular invasion of bone marrow tissue into the subchondral plate is often observed in articular cartilage, and the cells around the invaded vessel express high levels of MMP-3 (Shibakawa et al., 2005). MMP3 can disrupt the cell junctions between endothelial cells and degrade collagen within the ECM in the CNS, which facilitates the recruitment and accumulation of inflammatory cells (Behl et al., 2021). Compared with the CNS, articular cartilage lacks the protection of the blood-brain barrier; thus, inflammatory cells are more likely to infiltrate with the help of MMP3. MMP-3 can inhibit osteogenic differentiation and promote osteoclast maturity. Osteoclasts can resorb non-calcified and calcified cartilage independently of acidification. MMPs and cysteine proteases mediate the resorption of calcified cartilage in OA (Lofvall et al., 2018). Loss of cartilage matrix not only causes chondrocyte hypertrophy and apoptosis (Kim et al., 2001; Hwang and Kim, 2015) but also inhibits mesenchymal stem cell or osteoprogenitor cell differentiation into chondrocytes (Toh et al., 2016; Yin et al., 2016).

Regarding the symptoms of OA, mild synovitis is very common. Studies have shown that MMP-3 is not directly involved in the progression of synovitis, but the products of decomposing cartilage matrix, such as cartilage oligomeric matrix protein and damage-associated molecule patterns, can recruit inflammatory cells, leading to synovitis (Foell et al., 2007; Huang et al., 2015; Haraden et al., 2019). In addition, MMP-3 is highly expressed in synovial cells during the inflammatory response (Liao et al., 2020), so MMP-3 promotes synovitis and synovitis promotes the expression of MMP-3, forming a vicious cycle in the progression of OA.

Fibrosis, which is excessive accumulation and abnormal composition of ECM, often follow synovitis, and its occurrence and regression are closely related to the balance of MMPSTIMPs (Ko et al., 2019; Chen et al., 2021). Studies have found that MMP-3 is highly expressed in proliferative synovial tissues (Liao et al., 2020), and with the progression of fibrosis, 
the expression level of MMP-3 increases constantly, while the expression level of TIMP-1 decreases, leading to fibrosis regression (Hattori et al., 2017). The expression of MMP-3 only increases in the early stage of RA, a disease characterized by synovial hyperplasia and fibrosis, but a high expression of MMP3 is maintained throughout the course of OA; this may be one of the reasons that the pathological changes that occur in OA and RA are different.

Pain is the main symptom of OA that seriously affects patients' quality of life. Although MMP-3 has been reported to be involved in the development of peripheral neuropathy, researchers believe that MMP-3 is not directly related to pain in patients with OA (Fukushima et al., 2017). There is evidence that the effect of MSCs in the treatment of acute arthritis pain is not associated with specific changes in the synovial inflammatory cell population but instead is associated with the regulation of ADAMTS4, ADAMTS5, MMP3, and MMP9 expression in the synovial membrane after injury (Shu et al., 2020). However, no significant changes in MMP-3 levels have been observed in clinical studies on hyaluronic acid for the treatment of OA pain (Henrotin et al., 2019). Due to the lack of nerves in cartilage, patients do not experience significant pain in the early stage of OA. It has been confirmed that with the development of OA in cartilage, the subchondral fat pad (Heilmeier et al., 2020), subchondral bone and other structures are destroyed, resulting in the exposure or compression of nerve endings and thus intense pain. MMP-3 has been widely proven to be one of the major cytokines that damage the abovementioned structures (Belluzzi et al., 2019). Whether MMP-3 is directly related to pain in OA and whether it can be used as a therapeutic target for pain relief remain to be further studied.

\section{MECHANISM OF REGULATING MMP-3 EXPRESSION AND SECRETION IN OA}

The mechanism regulating the expression and secretion of MMP-3 in OA has been investigated for a long time. In this section, we show that MMP-3 is predominantly regulated by the inflammatory factors TNF- $\alpha$ and IL- $1 \beta$ in some classic pathways.

Interferon regulatory factor 5 (IRF5), TNF- $\alpha$ and MMP-3 expression levels were increased in cartilage cells from patients with OA. Further research confirmed that IRF5 can increase the inflammatory factors within the culture system in a dosedependent feedback regulatory mechanism in vitro (Lin et al., 2018). Both TNF- $\alpha$ and IL-1 $\beta$ can promote the synthesis and secretion of MMP-3 in preadipocytes (Gao and Bing, 2011). The expression of Kruppel-like factor 15 (KLF15) in chondrocytes was significantly reduced in patients with OA, while TNF- $\alpha$ treatment reduced p53-mediated KLF15 expression in human chondrocytes. Later studies confirmed that KLF15 can bind to the promoter of the MMP-3 gene and reduce TNF- $\alpha$-induced MMP3 expression at the transcriptional level (Yishuo et al., 2018).

\section{Nuclear Factor Kappa B Pathway}

Nuclear factor kappa B (NF- $\mathrm{B})$ enhancer binding protein is a nuclear transcriptional activator that binds to enhancer elements in many different cell types and can be activated by a variety of pathogenic stimuli, such as stress, cytokines, free radicals, heavy metals, ultraviolet radiation, oxidized LDL, and bacterial or viral antigens (Choi et al., 2019). As early as 2002, some researchers proved that MMP-1 and MMP-3 were constantly expressed in human macrophages, and such expression was dependent on the activation of NF- $\mathrm{B}$. By transfecting inhibitory NF- $\kappa \mathrm{B}$ subunits in cholesterol-induced rabbit foam cells, these scientists succeeded in reducing the expression levels of MMP$1 / 3$ (Chase et al., 2002). Later, researchers verified that NF$\kappa \mathrm{B}$ could interact with the promoter of the MMP-3 gene through chromatin immunoprecipitation (Souslova et al., 2010). Activation of the NF- $\mathrm{B}$ signaling pathway was found to be different between synovial cells and articular chondrocytes of OA patients, and the higher the level of RelA in the nucleus, the more MMP-3 expression was found in the cells (Ahmed et al., 2018).

\section{Mitogen-Activated Protein Kinase Pathway}

The Mitogen-activated protein kinase (MAPK) pathway has three levels of signaling: MAPK, MAPK kinase (MEK or $\mathrm{MKK}$ ), and the kinase of MAPK kinase (MEKK or MKKK). These three kinases can be activated in turn and together regulate many important physiological/pathological effects, such as cell growth, differentiation, stress and inflammatory responses. Some studies have confirmed that the MAPK pathway is involved in MMP-3 regulation in OA. By inhibiting MKK4 phosphorylation, miR-145 negatively regulates TNF- $\alpha$-mediated JNK and p38 activation, as well as the nuclear accumulation of p-c-Jun and p-ATF2, ultimately leading to alterations in gene transcription ( $\mathrm{Hu}$ et al., 2017). The levels of CX3CL1 in the synovium and serum are correlated with the incidence of OA. Further study proved that CX3CL1 increases MMP-3 levels by activating c-Raf, MEK, and ERK after binding with CX3CR1 (Hou et al., 2017).

\section{PI3K/Akt Pathway}

The PI3K/Akt pathway appears to positively and negatively regulate MMP-3 expression. In the field of pharmacological research, oroxylin A was reported to reduce the upregulation of MMP-3 and MMP-13 expression by inhibiting the IL$1 \beta$-induced activation of ERK1/2 and the PI3K/Akt signaling pathway (Zhang et al., 2021). Scoparone can prevent IL-1 $\beta$ from promoting inflammation by suppressing Akt phosphorylation and promoting NF- $\kappa$ B p65 phosphorylation, thus reducing MMP-3 expression during the progression of OA ( $\mathrm{Lu}$ et al., 2018). Another study suggested that moderate-intensity exercise can inhibit the inflammatory response of primary chondrocytes induced by IL-1 $\beta$ by increasing the level of 15-hydroxyeicosapetraenoic acid (15-HETE) in synovial fluid. The researchers found that 15-HETE could increase p-Akt levels and decrease the expression of MMP-3 and MMP-13 (Tian et al., 2021).

We described some pathways that are relevant to the regulatory mechanism of MMP-3 in OA (Figure 3). The 


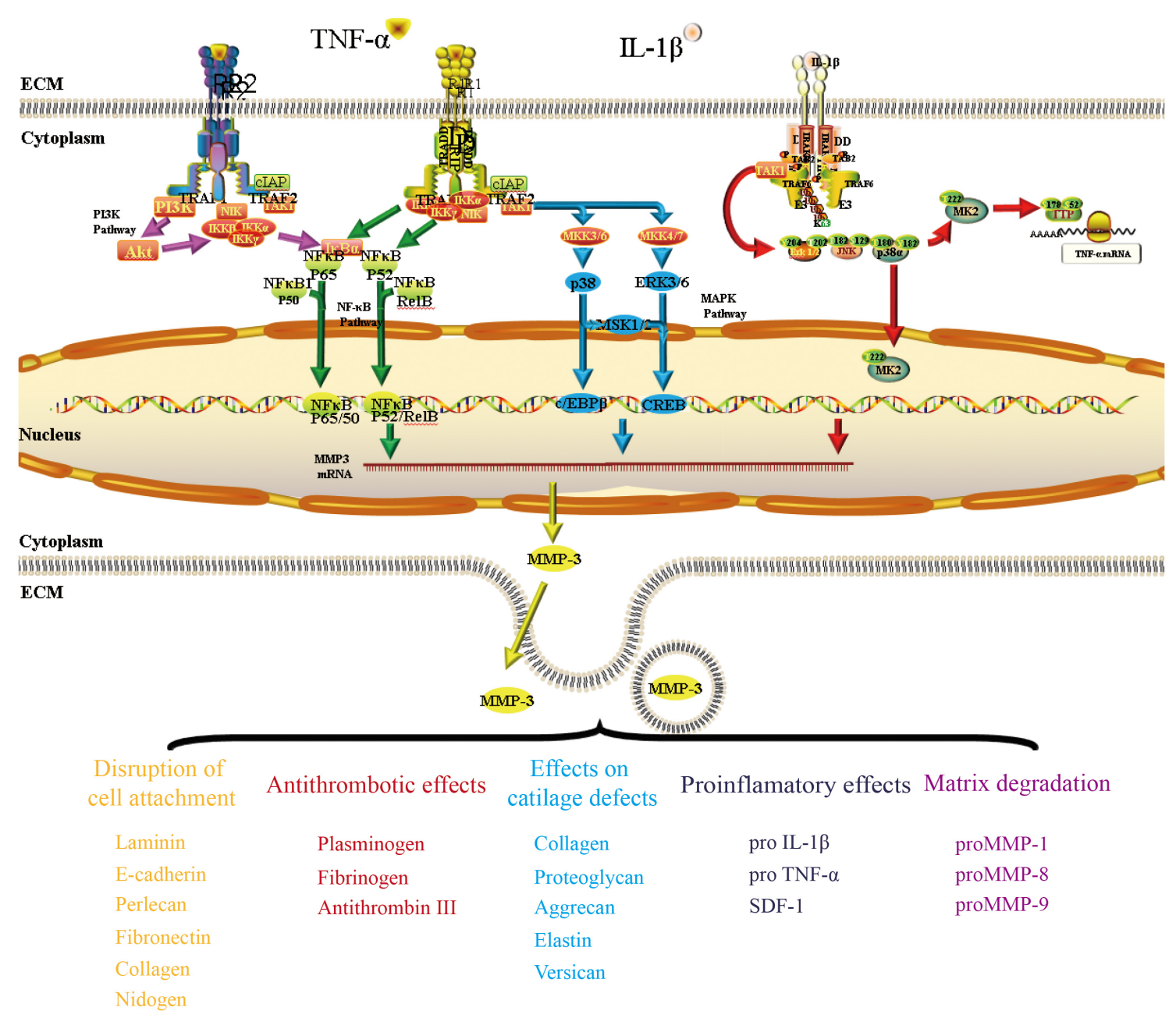

FIGURE 3 | The mechanism by which TNF- $\alpha$ and IL-1 $\beta$ regulate MMP-3 expression in OA. This figure presented three pathways (NF-kb pathway, MAPK pathway, and PI3K/Akt pathway) that have been mentioned a lot when discussing the regulation of MMP-3 expression. TNF- $\alpha$ can bind to its traditional receptor TNFRI or non-classic receptor TNFRII and pass the signal through both NF-kb pathway. The transcriptional factor NF-kb could bind to the promoter of MMP3 gene and regulate MMP-3 expression. The binding of TNF- $\alpha$ and its receptors could also activate the TAK 1 and then pass the signal through MAPK pathway. IL-1 $\beta$ could bind to its receptor and regulate MMP-3 expression through PI3K/Akt pathway.

complete mechanism is too complicated to be described in a short review, and further details of these pathways must be elucidated.

\section{CLINICAL DETECTION AND INHIBITORS OF MMP-3}

\section{MMP-3 Detection}

In inflammatory diseases involving bone and cartilage, such as rheumatoid arthritis (RA), ankylosing spondylitis (AS), and OA, the generally high level of serum MMP-3 suggests its potential as a diagnostic marker. Serum cartilage oligomeric protein and serum MMP-3 could be used as screening indicators to distinguish patients with knee arthritis from healthy people based on crosssectional studies (Tsvetoslav et al., 2018). Researchers also found that the MMP-3 mRNA level in the intervertebral synovial fluid of AS patients was higher than that of the healthy group (Liu et al., 2015). However, the expression level of MMP-3 also changes along with disease progression and remission, which suggests MMP-3 may be a marker of curative effects. Serum MMP-3 levels in patients with OA were significantly decreased after treatment with non-steroidal anti-inflammatory drugs (Efstathiou and Settas, 2017) and decreased significantly in RA patients treated with certolizumab pegol (Ling et al., 2017). Other studies in which RA patients were treated with methotrexate monotherapy (Kazuko et al., 2016) and AS patients were treated with etanercept (Dongyi et al., 2017) showed similar results to those described above. Based on this extensive evidence, researchers have confirmed that the MMP-3 level in serum and synovial fluid can be used as a marker in both preclinical and clinical situations.

The traditional method of detecting MMP-3 is individual enzyme-linked immunosorbent assays (ELISAs), which is 
inefficient and time-consuming. With the development of immunodetection methods, many new technologies have been exploited and used in MMP-3 detection. Bead-based immunosorbent assays are modifications of ELISAs in which the affinity peptide is linked with magnetic beads and coated on a platform. As the liquid sample flows across the platform, the target molecules are captured by the affinity peptides, and then, specific antibodies are added to detect the target molecules in a high-throughput manner (Adamcova and Simko, 2018). Reliable testing methods for MMP-3 based on this technology, such as the Human Luminex Screening Assay (10 analytes; Silverstein et al., 2017; Wyatt et al., 2019) and Myriad Human InflammationMAP ${ }^{\circledR} 1.0$ multiplex immunoassay (47 analytes; Haraden et al., 2019), have been developed. Biosensors based on metal-plasma resonance have extremely high sensitivity when detecting inflammatory factors; they are also a high-throughput method that can screen many samples at the same time (Huang et al., 2013), but the high cost limits their applications. Recently, fluorescent probe techniques were used to detect MMPs and ADAMTs in situ. Using fluorescently labeled peptides to bind MMPs and ADAMTs as probes after injection and then excite fluorescence in situ with a laser, researchers can observe MMP expression in arthrosis, not just serum and synovial fluid (Mohammadinejad et al., 2020). The improvement and development of MMP-3 detection has significantly promoted the clinical application of MMP-3. However, some researchers have noted that detection of full-length MMP-3 does not accurately reflect the progression of the disease. Probes targeting the active region of MMP-3 should be developed to accurately reflect the disease state by detecting the expression level and the activation degree of MMP-3 (Bernotiene et al., 2020). Therefore, further research in this field is needed.

\section{MMP-3 Inhibitors}

Researchers mainly inhibit MMP-3 expression through two methods, direct and indirect inhibition. First, by inhibiting TNF$\alpha$, IL-1 $\beta$ and its receptor, researchers can block the expression of MMP-3. Drugs corresponding to these mechanisms include anakinra, AMG 108 (a fully human monoclonal antibody to interleukin-1 receptor type 1), adalimumab, etanercept, and infliximab (Lespasio et al., 2017, 2018; Grassel and Muschter, 2020). Second, the transcription of MMP-3 can be directly blocked by miRNAs, including miRNA-155 (Korotkov et al., 2018) and miRNA-519d (Chengling et al., 2018), which have been experimentally tested. In addition, as a natural TIMP, TIMP-1 has an antagonistic function against MMP-3. However, due to its extensive and unpredictable side effects, this molecule cannot be applied in the clinic.

Because of their extensive anti-inflammatory effects, glucocorticoids are often used to control pain and relieve other symptoms in patients with RA, AS, and OA. Dexamethasone has been shown to inhibit the expression of endothelin in astrocytes, thereby reducing the synthesis of MMP-3 (Yutaka et al., 2017). Moreover, in traditional Chinese medicine, many botanicals have been proven to have multiple functions, such as anti-inflammatory, antioxidative, and antiapoptotic effects. Representative drugs include naringenin (Wang et al., 2017) or ginkgo biloba extracts (Zeng et al., 2015). In vitro experiments proved that these treatments can reduce the damage caused by MMP-3, but the specific mechanism is still unknown.

\section{DISCUSSION}

Matrix metalloproteinase 3 can show either positive or negative effects depending on its expression level and tissue specificity: the positive effects of MMP-3 play an important role in growth and development, especially the formation of some subtle structures, such as the early germ layer and maxillofacial bone. In adults, MMP-3 has been confirmed to promote axonal growth, cartilage ossification and adipose differentiation. The negative effects of MMP-3 are mostly related to the development of diseases. In the CNS, this molecule affects neurotrophy, causing axon damage, and neurodegeneration. In the cardiovascular system, MMP3 aggravates endothelial injury and promotes plaque shedding. In musculoskeletal system, this enzyme degrades the matrix of articular cartilage and destroys the original structure of joints, losing its ability to protect against stress. However, due to its large number of substrates and the broad expression of natural inhibitors, it is difficult to separately discuss the relationship between diseases and the biological functions of MMP-3. Therefore, this review summarized the relationship between MMP-3 and diseases, especially OA, based on the existing evidence.

Current studies generally have a standard method of researching MMP-3. First, these studies explore how gene defects or the pathological internal environment affects MMP3 expression. Second, researchers prove that MMP-3 expression is closely related to disease development and stop after these findings instead of investigating the MMP-3 interactions with specific cell components in physiological or pathological processes. MMP-3 has a variety of functions and causes obvious side effects when it is used as a therapeutic target. Elucidating the MMP cascade reaction initiated by MMP-3 or studying the reaction products of MMP-3 and its substrates can help us better understand the mechanism of action of MMP-3 so that we can use MMP-3 for clinical applications as soon as possible.

Matrix metalloproteinase 3 plays an important role in cell differentiation, especially in cell morphology and the ECM reshaping process. However, few studies have examined MMP-3 and stem cell-based tissue engineering. To date, most protocols for inducing stem cell differentiation include activating the inflammatory pathway, which can disrupt the homeostasis of stem cells and trigger the differentiation process. MMP-3 has both proinflammatory and anti-inflammatory effects. This molecule may be considered a target for regulating the differentiation of stem cells, as we did when researching $\mathrm{OA}$ and other inflammatory diseases related to MMP-3. In this review, we summarized the biofunctions of MMP-3 and its roles in cell differentiation and a variety of diseases, especially OA, hoping 
to draw more attention to MMP-3 and promote the study of its mechanisms.

\section{AUTHOR CONTRIBUTIONS}

JW, GZ, XL, XQ, JO, JD, and SM participated in the conception and writing of the manuscript. JD and SM reviewed and suggested modifications to the content. JD designed the structure

\section{REFERENCES}

Adamcova, M., and Simko, F. (2018). Multiplex biomarker approach to cardiovascular diseases. Acta Pharmacol. Sin. 39, 1068-1072. doi: 10.1038/aps. 2018.29

Ahmed, A. S., Gedin, P., Hugo, A., Bakalkin, G., Kanar, A., Hart, D. A., et al. (2018). Activation of NF-kappaB in Synovium versus Cartilage from Patients with Advanced Knee Osteoarthritis: A Potential Contributor to Inflammatory Aspects of Disease Progression. J. Immunol. 201, 1918-1927. doi: 10.4049/ jimmunol.1800486

Alexander, C. M., Selvarajan, S., Mudgett, J., and Werb, Z. (2001). Stromelysin1 regulates adipogenesis during mammary gland involution. J. Cell Biol. 152, 693-703. doi: 10.1083/jcb.152.4.693

Andries, L., Van Hove, I., Moons, L., and De Groef, L. (2017). Matrix Metalloproteinases During Axonal Regeneration, a Multifactorial Role from Start to Finish. Mol. Neurobiol. 54, 2114-2125. doi: 10.1007/s12035-016-9801-x

Barkho, B. Z., Munoz, A. E., Li, X., Li, L., Cunningham, L. A., and Zhao, X. (2008). Endogenous Matrix Metalloproteinase (MMP)-3 and MMP-9 Promote the Differentiation and Migration of Adult Neural Progenitor Cells in Response to Chemokines. Stem Cells 26, 3139-3149. doi: 10.1634/stemcells.2008-0519

Behl, T., Kaur, G., Sehgal, A., Bhardwaj, S., Singh, S., Buhas, C., et al. (2021). Multifaceted Role of Matrix Metalloproteinases in Neurodegenerative Diseases: Pathophysiological and Therapeutic Perspectives. Int. J. Mol. Sci. 22:1413. doi: $10.3390 /$ ijms 22031413

Behrendt, P., Preusse-Prange, A., Kluter, T., Haake, M., Rolauffs, B., Grodzinsky, A. J., et al. (2016). IL-10 reduces apoptosis and extracellular matrix degradation after injurious compression of mature articular cartilage. Osteoarthr. Cartil. 24, 1981-1988. doi: 10.1016/j.joca.2016.06.016

Belluzzi, E., Olivotto, E., Toso, G., Cigolotti, A., Pozzuoli, A., Biz, C., et al. (2019). Conditioned media from human osteoarthritic synovium induces inflammation in a synoviocyte cell line. Connect Tissue Res. 60, 136-145. doi: 10.1080/ 03008207.2018.1470167

Bernotiene, E., Bagdonas, E., Kirdaite, G., Bernotas, P., Kalvaityte, U., Uzieliene, I., et al. (2020). Emerging Technologies and Platforms for the Immunodetection of Multiple Biochemical Markers in Osteoarthritis Research and Therapy. Front. Med. 7:572977. doi: 10.3389/fmed.2020.572977

Carine, C., Bernard, M., Marie-Noëlle, M., Stéphanie, B., Patrick, A., Emmanuel, V. O., et al. (2003). Matrix Metalloproteinases Are Differentially Expressed in Adipose Tissue during Obesity and Modulate Adipocyte Differentiation. J. Biol. Chem. 278, 11888-11896. doi: 10.1074/jbc.m209196200

Chase, A. J., Bond, M., Crook, M. F., and Newby, A. C. (2002). Role of nuclear factor-kappa B activation in metalloproteinase- $1,-3$, and -9 secretion by human macrophages in vitro and rabbit foam cells produced in vivo. Arterioscler. Thromb. Vasc. Biol. 22, 765-771. doi: 10.1161/01.atv.0000015078.09208.92

Chen, C. L., Zhang, L., Jiao, Y. R., Zhou, Y., Ge, Q. F., Li, P. C., et al. (2019). miR134 inhibits osteosarcoma cell invasion and metastasis through targeting MMP1 and MMP3 in vitro and in vivo. FEBS Lett. 593, 1089-1101. doi: 10.1002/18733468.13387

Chen, J., Liu, Z., Wang, H., Qian, L., Li, Z., Song, Q., et al. (2021). SIRT6 enhances telomerase activity to protect against DNA damage and senescence in hypertrophic ligamentum flavum cells from lumbar spinal stenosis patients. Aging 13, 6025-6040. doi: 10.18632/aging.202536

Chen, Q., Jin, M., Yang, F., Zhu, J., Xiao, Q., and Zhang, L. (2013). Matrix metalloproteinases: inflammatory regulators of cell behaviors in vascular formation and remodeling. Mediat. Inflamm. 2013:928315. of review manuscript. All authors contributed to the article and approved the submitted version.

\section{FUNDING}

This study was financially supported by the National Key R\&D Program of China (2017YFC1105000).

Chengling, C., Xin, L., Xue, B., Tong, Z., Mengxue, W., Ranchen, X., et al. (2018). MiR-519d suppresses breast cancer tumorigenesis and metastasis via targeting MMP3. Int. J. Biol. Sci. 14, 228-236. doi: 10.7150/ijbs.22849

Chin, J. R., and Werb, Z. (1997). Matrix metalloproteinases regulate morphogenesis, migration and remodeling of epithelium, tongue skeletal muscle and cartilage in the mandibular arch. Development 124, 1519-1530. doi: 10.1242/dev.124.8.1519

Choi, M. C., Jo, J., Park, J., Kang, H. K., and Park, Y. (2019). NF-kappaB Signaling Pathways in Osteoarthritic Cartilage Destruction. Cells 8:734. doi: 10.3390/ cells 8070734

Cross, M., Smith, E., Hoy, D., Nolte, S., Ackerman, I., Fransen, M., et al. (2014). The global burden of hip and knee osteoarthritis: estimates from the global burden of disease 2010 study. Ann. Rheum. Dis. 73, 1323-1330. doi: 10.1136/ annrheumdis-2013-204763

Cui, N., Hu, M., and Khalil, R. A. (2017). Biochemical and Biological Attributes of Matrix Metalloproteinases. Prog. Mol. Biol. Transl. Sci. 147, 1-73. doi: 10.1016/ bs.pmbts.2017.02.005

De Groef, L., Andries, L., Lemmens, K., Van Hove, I., and Moons, L. (2015). Matrix metalloproteinases in the mouse retina: a comparative study of expression patterns and MMP antibodies. BMC Ophthalmol. 15:187. doi: 10.1186/s12886015-0176-y

Dongyi, H., Qi, Z., Quan, Z., Qing, Q., Hongmei, S., Liza, Z. M., et al. (2017). Correlation of serum MMP3 and other biomarkers with clinical outcomes in patients with ankylosing spondylitis: a pilot study. Clin. Rheumatol. 36, 1819-1826. doi: 10.1007/s10067-017-3624-7

Efstathiou, M., and Settas, L. (2017). The effect of non-steroidal anti-inflammatory drugs on matrix metalloproteinases levels in patients with osteoarthritis. Mediterr. J. Rheumatol. 28, 133-141. doi: 10.31138/mjr.28.3.133

Eguchi, T., Kubota, S., Kawata, K., Mukudai, Y., Uehara, J., Ohgawara, T., et al. (2008). Novel transcription-factor-like function of human matrix metalloproteinase 3 regulating the CTGF/CCN2 gene. Mol. Cell Biol. 28, 23912413. doi: $10.1128 / \mathrm{mcb} .01288-07$

Foell, D., Wittkowski, H., and Roth, J. (2007). Mechanisms of disease: a 'DAMP' view of inflammatory arthritis. Nat. Clin. Pract. Rheumatol. 3, 382-390. doi: 10.1038/ncprheum0531

Franses, R. E., McWilliams, D. F., Mapp, P. I., and Walsh, D. A. (2010). Osteochondral angiogenesis and increased protease inhibitor expression in OA. Osteoarthr. Cartilage 18, 563-571. doi: 10.1016/j.joca.2009.11.015

Fukushima, K., Inoue, G., Fujimaki, H., Uchida, K., Miyagi, M., Nagura, N., et al. (2017). The cytokine expression in synovial membrane and the relationship with pain and pathological findings at hip arthroscopy. J. Exp. Orthop. 4:12.

Gao, D., and Bing, C. (2011). Macrophage-induced expression and release of matrix metalloproteinase 1 and 3 by human preadipocytes is mediated by IL-1beta via activation of MAPK signaling. J. Cell Physiol. 226, 2869-2880. doi: 10.1002/jcp. 22630

Ghaffarpour, S., Ghazanfari, T., Kabudanian Ardestani, S., Pourfarzam, S., Fallahi, F., Shams, J., et al. (2017). Correlation between MMP-9 and MMP-9/ TIMPs Complex with Pulmonary Function in Sulfur Mustard Exposed Civilians: Sardasht-Iran Cohort Study. Arch. Iran Med. 20, 74-82.

Grassel, S., and Muschter, D. (2020). Recent advances in the treatment of osteoarthritis. F1000Res. 9:F1000FacultyRev-325.

Guizani, I., Zidi, W., Zayani, Y., Boudiche, S., Hadj-Taieb, S., Sanhaji, H., et al. (2019). Matrix metalloproteinase-3 predicts clinical cardiovascular outcomes in patients with coronary artery disease: a 5 years cohort study. Mol. Biol. Rep. 46, 4699-4707. doi: 10.1007/s11033-019-04914-4 
Haraden, C. A., Huebner, J. L., Hsueh, M. F., Li, Y. J., and Kraus, V. B. (2019). Synovial fluid biomarkers associated with osteoarthritis severity reflect macrophage and neutrophil related inflammation. Arthritis Res. Ther. 21:146.

Hattori, T., Itoh, S., and Takigawa, M. (2017). Generation and Analysis of CartilageSpecific CCN2 Overexpression in Transgenic Mice. Methods Mol. Biol. 1489, 391-403. doi: 10.1007/978-1-4939-6430-7_32

He, Z., Nie, P., Lu, J., Ling, Y., Guo, J., Zhang, B., et al. (2020). Less mechanical loading attenuates osteoarthritis by reducing cartilage degeneration, subchondral bone remodelling, secondary inflammation, and activation of NLRP3 inflammasome. Bone Joint Res. 9, 731-741. doi: 10.1302/ 2046-3758.910.bjr-2019-0368.r2

Heilmeier, U., Mamoto, K., Amano, K., Eck, B., Tanaka, M., Bullen, J. A., et al. (2020). Infrapatellar fat pad abnormalities are associated with a higher inflammatory synovial fluid cytokine profile in young adults following ACL tear. Osteoarthr. Cartilage 28, 82-91. doi: 10.1016/j.joca.2019.09.001

Henrotin, Y., Bannuru, R., Malaise, M., Ea, H. K., Confavreux, C., Bentin, J., et al. (2019). Hyaluronan derivative HYMOVIS(R) increases cartilage volume and type ii collagen turnover in osteoarhritic knee: data from MOKHA study. BMC Musculoskelet Disord. 20:293. doi: 10.1186/s12891-019-2667-0

Hong, S., Kang, J. K., Park, J. J., Ryu, E. S., Choi, S. S., Lee, S. H., et al. (2010). Association of Matrix metalloproteinase-3 with cardiogenic activity during Noggin-induced differentiation of mouse embryonic stem cells. Int. J. Cardiol. 141, 49-60. doi: 10.1016/j.ijcard.2008.11.156

Hou, S. M., Hou, C. H., and Liu, J. F. (2017). CX3CL1 promotes MMP-3 production via the CX3CR1, c-Raf, MEK, ERK, and NF-kappaB signaling pathway in osteoarthritis synovial fibroblasts. Arthritis Res. Ther. 19:282. doi: 10.1016/j. biopha.2018.05.128

Hu, G., Zhao, X., Wang, C., Geng, Y., Zhao, J., Xu, J., et al. (2017). MicroRNA-145 attenuates TNF-alpha-driven cartilage matrix degradation in osteoarthritis via direct suppression of MKK4. Cell Death Dis. 8:e3140. doi: 10.1038/cddis.2017. 522

Hu, W., Ye, Y., Yin, Y., Sang, P., Li, L., Wang, J., et al. (2018). Association of matrix metalloprotease 1,3 , and 12 polymorphisms with rheumatic heart disease in a Chinese Han population. BMC Med. Genet. 19:27. doi: 10.1186/s12881-0180538-4

Huang, H., Tohme, S., Al-Khafaji, A. B., Tai, S., Loughran, P., Chen, L., et al. (2015). Damage-associated molecular pattern-activated neutrophil extracellular trap exacerbates sterile inflammatory liver injury. Hepatology 62, 600-614. doi: 10.1002/hep. 27841

Huang, J. F., Du, W. X., and Chen, J. J. (2016). Elevated expression of matrix metalloproteinase-3 in human osteosarcoma and its association with tumor metastasis. J. BUON. 21, 235-243.

Huang, X. Y., Han, L. Y., Huang, X. D., Guan, C. H., Mao, X. L., and Ye, Z. S. (2017). Association of Matrix Metalloproteinase-1 and Matrix Metalloproteinase-3 Gene Variants with Ischemic Stroke and Its Subtype. J. Stroke Cerebrovasc. Dis. 26, 368-375. doi: 10.1016/j.jstrokecerebrovasdis.2016.09.034

Huang, X. Y., Han, L. Y., Huang, X. D., Guan, C. H., Mao, X. L., and Ye, Z. S. (2016). Impact of $5 \mathrm{~A} / 6 \mathrm{~A}$ polymorphism of matrix metalloproteinase- 3 on recurrent atherosclerotic ischemic stroke in Chinese. Int. J. Neurosci. 126, 936-941. doi: 10.3109/00207454.2015.1088013

Huang, Y. C., Chiang, C. Y., Li, C. H., Chang, T. C., Chiang, C. S., Chau, L. K., et al. (2013). Quantification of tumor necrosis factor-alpha and matrix metalloproteinases- 3 in synovial fluid by a fiber-optic particle plasmon resonance sensor. Analyst 138, 4599-4606. doi: 10.1039/c3an00276d

Husslein, H., Haider, S., Meinhardt, G., Prast, J., Sonderegger, S., and Knofler, M. (2009). Expression, regulation and functional characterization of matrix metalloproteinase-3 of human trophoblast. Placenta 30, 284-291. doi: 10.1016/ j.placenta.2008.12.002

Hwang, H. S., and Kim, H. A. (2015). Chondrocyte Apoptosis in the Pathogenesis of Osteoarthritis. Int. J. Mol. Sci. 16, 26035-26054. doi: 10.3390/ijms161125943

Inge, V. H., Kim, L., Sarah, V. D. V., Mieke, V., and Lieve, M. (2012). Matrix metalloproteinase- 3 in the central nervous system: a look on the bright side. J. Neurochem. 123, 203-216. doi: 10.1111/j.1471-4159.2012.07900.x

Jiajia, Z., Xin, Z., Qingming, T., Ran, Y., Shaoling, Y., Yanlin, L., et al. (2018). BMAL1 Deficiency Contributes to Mandibular Dysplasia by Upregulating MMP3. Stem Cell Rep. 10, 180-195. doi: 10.1016/j.stemcr.2017.11.017

Kato, T., Miyaki, S., Ishitobi, H., Nakamura, Y., Nakasa, T., Lotz, M. K., et al. (2014). Exosomes from IL-1beta stimulated synovial fibroblasts induce osteoarthritic changes in articular chondrocytes. Arthritis. Res. Ther. 16:R163.

Kazuko, S., Takashi, Y., Miki, M., Ryosuke, Y., Ken, T., Shigeaki, I., et al. (2016). MMP-3 as a predictor for structural remission in RA patients treated with MTX monotherapy. Arthritis Res. Therapy 18:55.

Kim, H. A., Suh, D. I., and Song, Y. W. (2001). Relationship between chondrocyte apoptosis and matrix depletion in human articular cartilage. J. Rheumatol. 28, 2038-2045.

Ko, J. H., Kang, Y. M., Yang, J. H., Kim, J. S., Lee, W. J., Kim, S. H., et al. (2019). Regulation of MMP and TIMP expression in synovial fibroblasts from knee osteoarthritis with flexion contracture using adenovirus-mediated relaxin gene therapy. Knee 26, 317-329. doi: 10.1016/j.knee.2019.01.010

Korotkov, A., Broekaart, D. W. M., van Scheppingen, J., Anink, J. J., Baayen, J. C., Idema, S., et al. (2018). Increased expression of matrix metalloproteinase 3 can be attenuated by inhibition of microRNA-155 in cultured human astrocytes. J. Neuroinflamm. 15:211.

Koyama, K., Ohba, T., Odate, T., Wako, M., and Haro, H. (2020). Pathological features of established osteoarthritis with hydrathrosis are similar to rheumatoid arthritis. Clin. Rheumatol. 40, 2007-2012. doi: 10.1007/s10067020-05453-1

Lee, H. R., Shon, O. J., Park, S. I., Kim, H. J., Kim, S., Ahn, M. W., et al. (2016). Platelet-Rich Plasma Increases the Levels of Catabolic Molecules and Cellular Dedifferentiation in the Meniscus of a Rabbit Model. Int. J. Mol. Sci. 17:120. doi: 10.3390/ijms17010120

Lespasio, M. J., Piuzzi, N. S., Husni, M. E., Muschler, G. F., Guarino, A., and Mont, M. A. (2017). Knee Osteoarthritis: A Primer. Perm. J. 21, 16-183.

Lespasio, M. J., Sultan, A. A., Piuzzi, N. S., Khlopas, A., Husni, M. E., Muschler, G. F., et al. (2018). Hip Osteoarthritis: A Primer. Perm. J. 22, 17-84.

Liao, L., Zhang, S., Zhao, L., Chang, X., Han, L., Huang, J., et al. (2020). Acute Synovitis after Trauma Precedes and is Associated with Osteoarthritis Onset and Progression. Int. J. Biol. Sci. 16, 970-980. doi: 10.7150/ijbs.39015

Lijnen, H. R. (2002). Matrix metalloproteinases and cellular fibrinolytic activity. Biochemistry 67, 92-98.

Lin, G., Ruihu, H., Fengde, T., Ning, A., and Kunzheng, W. (2018). Interferon regulatory factor 5 (IRF5) regulates the expression of matrix metalloproteinase3 (MMP-3) in human chondrocytes. Int. Immunopharmacol. 55, 231-236. doi: 10.1016/j.intimp.2017.11.035

Ling, Z., Geng, W., Xin, L., Jing, S., Ling, C., and Huji, X. (2017). Matrix metalloproteinase- 3 and the 7 -joint ultrasound score in the assessment of disease activity and therapeutic efficacy in patients with moderate to severe rheumatoid arthritis. Arthritis Res. Therapy 19:250.

Liu, K. G., He, Q. H., Tan, J. W., and Liao, G. J. (2015). Expression of TNF- $\alpha$, VEGF, and MMP-3 mRNAs in synovial tissues and their roles in fibroblast-mediated osteogenesis in ankylosing spondylitis. Genet. Mol. Res. 14, 6852-6858. doi: 10.4238/2015.june. 18.28

Lofvall, H., Newbould, H., Karsdal, M. A., Dziegiel, M. H., Richter, J., Henriksen, K., et al. (2018). Osteoclasts degrade bone and cartilage knee joint compartments through different resorption processes. Arthritis Res. Ther. 20:67.

Lu, C., Li, Y., Hu, S., Cai, Y., Yang, Z., and Peng, K. (2018). Scoparone prevents IL-1beta-induced inflammatory response in human osteoarthritis chondrocytes through the PI3K/Akt/NF-kappaB pathway. Biomed. Pharmacother. 106, 11691174. doi: 10.1016/j.biopha.2018.07.062

Ma, Y., Cang, S., Li, G., Su, Y., Zhang, H., Wang, L., et al. (2019). Integrated analysis of transcriptome data revealed MMP3 and MMP13 as critical genes in anaplastic thyroid cancer progression. J. Cell. Physiol. 234, 22260-22271. doi: $10.1002 /$ jcp. 28793

Mohammadinejad, R., Ashrafizadeh, M., Pardakhty, A., Uzieliene, I., Denkovskij, J., Bernotiene, E., et al. (2020). Nanotechnological Strategies for Osteoarthritis Diagnosis, Monitoring, Clinical Management, and Regenerative Medicine: Recent Advances and Future Opportunities. Curr. Rheumatol. Rep. 22:12.

Nagase, H., Visse, R., and Murphy, G. (2006). Structure and function of matrix metalloproteinases and TIMPs. Cardiovasc. Res. 69, 562-573. doi: 10.1016/j. cardiores.2005.12.002

Niada, S., Giannasi, C., Gomarasca, M., Stanco, D., Casati, S., and Brini, A. T. (2019). Adipose-derived stromal cell secretome reduces TNFalpha-induced hypertrophy and catabolic markers in primary human articular chondrocytes. Stem Cell Res. 38:101463. doi: 10.1016/j.scr.2019.101463 
Nishida, K., Kuchiiwa, S., Oiso, S., Futagawa, T., Masuda, S., Takeda, Y., et al. (2008). Up-regulation of matrix metalloproteinase-3 in the dorsal root ganglion of rats with paclitaxel-induced neuropathy. Cancer Sci. 99, 1618-1625. doi: 10.1111/j.1349-7006.2008.00877.x

Otto, T. C., Bowers, R. R., and Lane, M. D. (2007). BMP-4 treatment of C3H10T1/2 stem cells blocks expression of MMP-3 and MMP-13. Biochem. Biophys. Res. Commun. 353, 1097-1104. doi: 10.1016/j.bbrc.2006.12.170

Pinto, A. F., Terra, R. M., Guimaraes, J. A., Kashiwagi, M., Nagase, H., Serrano, S. M., et al. (2006). Structural features of the reprolysin atrolysin C and tissue inhibitors of metalloproteinases (TIMPs) interaction. Biochem. Biophys. Res. Commun. 347, 641-648. doi: 10.1016/j.bbrc.2006.06.143

Shibakawa, A., Yudoh, K., Masuko-Hongo, K., Kato, T., Nishioka, K., and Nakamura, H. (2005). The role of subchondral bone resorption pits in osteoarthritis: MMP production by cells derived from bone marrow. Osteoarthr. Cartilage 13, 679-687. doi: 10.1016/j.joca.2005.04.010

Shu, C. C., Zaki, S., Ravi, V., Schiavinato, A., Smith, M. M., and Little, C. B. (2020). The relationship between synovial inflammation, structural pathology, and pain in post-traumatic osteoarthritis: differential effect of stem cell and hyaluronan treatment. Arthritis Res. Ther. 22:29.

Silverstein, A. M., Stoker, A. M., Ateshian, G. A., Bulinski, J. C., Cook, J. L., and Hung, C. T. (2017). Transient expression of the diseased phenotype of osteoarthritic chondrocytes in engineered cartilage. J. Orthop. Res. 35, 829-836. doi: 10.1002/jor.23301

Souslova, V., Townsend, P. A., Mann, J., van der Loos, C. M., Motterle, A., D'Acquisto, F., et al. (2010). Allele-specific regulation of matrix metalloproteinase-3 gene by transcription factor NFkappaB. PLoS One 5:e9902. doi: 10.1371/journal.pone.0009902

Tian, Y., Gou, J., Zhang, H., Lu, J., Jin, Z., Jia, S., et al. (2021). The antiinflammatory effects of 15-HETE on osteoarthritis during treadmill exercise. Life Sci. 273:119260. doi: 10.1016/j.lfs.2021.119260

Toh, W. S., Foldager, C. B., Hui, J. H., Olsen, B. R., and Spector, M. (2016). Exploiting Stem Cell-Extracellular Matrix Interactions for Cartilage Regeneration: A Focus on Basement Membrane Molecules. Curr. Stem Cell Res. Ther. 11, 618-625. doi: 10.2174/1574888x10666150723150525

Tong, Z., Liu, Y., Chen, B., Yan, L., and Hao, D. (2017). Association between MMP3 and TIMP3 polymorphisms and risk of osteoarthritis. Oncotarget 8, 83563-83569. doi: 10.18632/oncotarget.18745

Tsvetoslav, G., Mariana, I., Aleksandar, K., Tsvetelina, V., Asen, M., Ekaterina, K., et al. (2018). Cartilage oligomeric protein, matrix metalloproteinase-3, and Coll2-1 as serum biomarkers in knee osteoarthritis: a cross-sectional study. Rheumatol. Int. 38, 821-830. doi: 10.1007/s00296-017-3887-y

Wang, C. C., Guo, L., Tian, F. D., An, N., Luo, L., Hao, R. H., et al. (2017). Naringenin regulates production of matrix metalloproteinases in the kneejoint and primary cultured articular chondrocytes and alleviates pain in rat osteoarthritis model. Braz. J. Med. Biol. Res. 50:e5714.

Woo, C. H., Kim, H. K., Jung, G. Y., Jung, Y. J., Lee, K. S., Yun, Y. E., et al. (2020). Small extracellular vesicles from human adipose-derived stem cells attenuate cartilage degeneration. J. Extracell Vesicles 9:1735249. doi: 10.1080/20013078. 2020.1735249

Wyatt, L. A., Nwosu, L. N., Wilson, D., Hill, R., Spendlove, I., Bennett, A. J., et al. (2019). Molecular expression patterns in the synovium and their association with advanced symptomatic knee osteoarthritis. Osteoarthritis Cartilage 27, 667-675. doi: 10.1016/j.joca.2018.12.012

Yamada, Y., Nakamura-Yamada, S., Umemura-Kubota, E., and Baba, S. (2019). Diagnostic Cytokines and Comparative Analysis Secreted from Exfoliated Deciduous Teeth, Dental Pulp, and Bone Marrow Derived Mesenchymal Stem Cells for Functional Cell-Based Therapy. Int. J. Mol. Sci. 20:5900. doi: 10.3390/ ijms 20235900

Yin, H., Wang, Y., Sun, Z., Sun, X., Xu, Y., Li, P., et al. (2016). Induction of mesenchymal stem cell chondrogenic differentiation and functional cartilage microtissue formation for in vivo cartilage regeneration by cartilage extracellular matrix-derived particles. Acta Biomater. 33, 96-109. doi: 10.1016/ j.actbio.2016.01.024

Yishuo, L., Min, Z., and Weiguo, X. (2018). KLF15 Regulates the Expression of MMP-3 in Human Chondrocytes. J. Interferon Cytokine Res. 38, 356-362. doi: 10.1089/jir.2017.0135

Yutaka, K., Ayano, U., Kana, A., Kuniaki, I., Shogo, T., Keisuke, T., et al. (2017). Dexamethasone Downregulates Endothelin Receptors and Reduces Endothelin-Induced Production of Matrix Metalloproteinases in Cultured Rat Astrocytes. Mol. Pharmacol. 92, 57-66. doi: 10.1124/mol.116.10 7300

Zeng, J. Z., Ma, L. F., Meng, H., Yu, H. M., Zhang, Y. K., and Guo, A. (2015). Effect of Ginkgo biloba extract on matrix metalloproteinase-3 expression in a rat model of chondrocyte injury. Genet. Mol. Res. 14, 18280-18286. doi: 10.4238/2015.december.23.15

Zhang, Y., Weng, Q., Chen, J., Li, M., and Han, J. (2021). Oroxylin A attenuates IL1beta-induced inflammatory reaction via inhibiting the activation of the ERK and PI3K/AKT signaling pathways in osteoarthritis chondrocytes. Exp. Ther. Med. 21:388.

Zheng, J., Zhou, Y., Li, X. J., and Hu, J. M. (2019). MiR-574-3p exerts as a tumor suppressor in ovarian cancer through inhibiting MMP3 expression. Eur. Rev. Med. Pharmacol. Sci. 23, 6839-6848.

Conflict of Interest: The authors declare that the research was conducted in the absence of any commercial or financial relationships that could be construed as a potential conflict of interest.

Copyright (® 2021 Wan, Zhang, Li, Qiu, Ouyang, Dai and Min. This is an openaccess article distributed under the terms of the Creative Commons Attribution License (CC BY). The use, distribution or reproduction in other forums is permitted, provided the original author(s) and the copyright owner(s) are credited and that the original publication in this journal is cited, in accordance with accepted academic practice. No use, distribution or reproduction is permitted which does not comply with these terms. 